\title{
Sensor Interaction as a Source of the Electromagnetic Field Measurement Error
}

\author{
R. Hartansky, V. Smiesko, M. Bittera, L. Marsalka, O. Cicakova, L. Letanovska \\ Institute of Electrical Engineering, Faculty of Electrical Engineering and Information Technology, \\ Slovak University of Technology, Ilkovicova 3, 81219 Bratislava, Slovakia, \\ rene.hartansky@stuba.sk
}

\begin{abstract}
The article deals with analytical calculation and numerical simulation of interactive influence of electromagnetic sensors. Sensors are components of field probe, whereby their interactive influence causes the measuring error. Electromagnetic field probe contains three mutually perpendicular spaced sensors in order to measure the vector of electrical field. Error of sensors is enumerated with dependence on interactive position of sensors. Based on that, proposed were recommendations for electromagnetic field probe construction to minimize the sensor interaction and measuring error.
\end{abstract}

Keywords: Electromagnetic fields, isotropic sensor, mutual impedance, measurement field error.

\section{INTRODUCTION}

$I_{n}^{N}$ N MANY areas of electrical engineering it is required to measure the amplitude of electromagnetic field with sufficient accuracy (e.g. during electromagnetic compatibility testing of electrical devices). The measurement is in many cases performed in a far field. Then it is sufficient to measure only one component (electric) of the electromagnetic field while the other (magnetic) can be easily estimated. Despite this simplification, the measured component of the electromagnetic field is a vector. This means that we need to know all the vector components ( $\mathrm{x}, \mathrm{y}$, z) which determine its final amplitude. To measure the intensity of all three vector components of electric (magnetic) field, three field sensors placed on the $\mathrm{x}, \mathrm{y}$, and $\mathrm{z}$ axis of the coordinate system have to be used. Or they can create an omnidirectional probe of the electromagnetic field. In general, such a probe is made of three orthogonal sensors and the output voltage of the probe is determined as follows:

$$
U_{E}=\sqrt{U_{x}^{2}+U_{y}^{2}+U_{z}^{2}}
$$

In order to ensure sufficient accuracy, the sensors have to meet a series of conditions: frequency independence [1]; sufficient output voltage [1]; sensitivity to only one component of the electric field vector [1], [2]; linearity [2], [5]; no (or negligible) interaction with surrounding objects. In the scientific literature, attention is given to the first four conditions; however, the condition of minimal interaction is not solved. Therefore, this article deals with the mutual interaction of sensors and their effects on the error of the electromagnetic field measurement.

\section{ELECTROMAGNETIC FIELD AND SENSOR INFLUENCE}

Let us assume that the field sensor - dipole antenna in homogeneous electromagnetic field (incidence field) which has the vector of $\mathrm{E}$ component of electromagnetic field oriented in the $z$ axis direction is placed. The sensor is tangentially oriented to the field E. If the dipole antenna is on the resonance with incidence frequency then the terminal voltage $\mathrm{U}_{\mathrm{so}}$ on unloaded dipole can be calculated as [5].

$$
U_{s o}=h_{e f} \cdot E_{i t}
$$

Whereby $h_{e f}$ is effective dipole length and $E_{i t}$ is the magnitude of tangential electric field component. Halflength dipole $\mathrm{h}_{\mathrm{ef}}$ can be calculated by [5]

$$
h_{e f}=\frac{2(1-\cos (k h))}{k}
$$

Where $k=2 \pi / \lambda$ is a wave number, $h$ is half-length of dipole, $\lambda$ is wavelength of intruding electromagnetic field. If the dipole antenna is loaded with terminal impedance $Z_{\mathrm{s}}$, the terminal voltage of loaded dipole is:

$$
U_{s L}=U_{s o} \frac{Z_{s}}{Z_{i}+Z_{s}}
$$

$\mathrm{Z}_{i}$ is inner impedance of dipole. From the equation (4) terminal dipole current is obtained

$$
I_{s L}=U_{s o} \frac{1}{Z_{i}+Z_{s}}
$$

The current flowing through the electric field sensor (through dipole) creates radiated electromagnetic field - its components are both tangential and radial to the position of the sensor.

The magnitude of radiated field strongly decays with the distance. In the vicinity of sensor, the component $E_{y}$ is formed. Its magnitude is calculated in [4] by

$$
E_{y r}=-\frac{30 j I_{s L} y}{x^{2}+y^{2}}\left\{-\frac{e^{-j k R_{1}}}{R_{1}}-\frac{e^{-j k R_{2}}}{R_{2}}+\frac{e^{-j k r}}{r} \cos (k h)\right\}
$$


$\mathrm{R}_{1}, \mathrm{R}_{2}$ and $\mathrm{r}$ represent the magnitudes of position vectors. Their meaning is interpreted in Fig. 1.

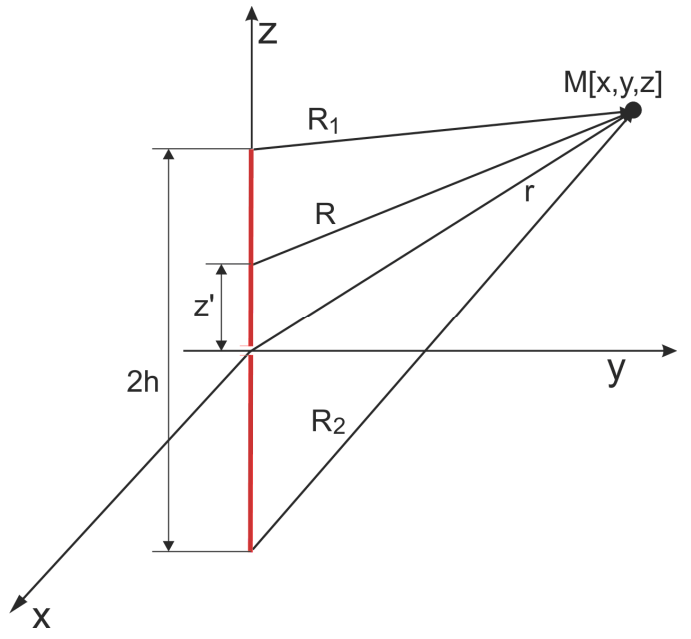

Fig.1. Position vectors of the dipole.

The original incidence field is influenced by the presence of the field radiated by dipole. There are components formed which are not proper to the original field but to the influence of the sensor to its close neighborhood.

Now, see the beginning of this article. If the electromagnetic field probe consists of three field sensors sensing the electric components $\mathrm{E}_{\mathrm{x}}, \mathrm{E}_{\mathrm{y}}$, and $\mathrm{E}_{\mathrm{z}}$, then according to the original assumption (the existence of $\mathrm{E}_{\mathrm{z}}$ component only), the output voltage of individual transducers has to be $\mathrm{U}_{\mathrm{x}}=\mathrm{U}_{\mathrm{y}}=0$. Actually, it is not, because influence of the very existence of sensors (dipoles) in the electric field has formed the components $\mathrm{E}_{\mathrm{xr}}$ and $\mathrm{E}_{\mathrm{yr}}$. Under the influence of that physical coupling, the measurement is affected and the error is brought into the measurement results.

This part of the article deals with the calculation of the change of the probe output voltage caused by the mutual coupling between sensors. To simplify the solution, the content of only two sensors $Z$ and $Y$ in the probe is assumed. The electric field component is oriented only into one direction, for example in the $z$ direction. Because the sensors $\mathrm{Z}$ and $\mathrm{Y}$ are placed close to each other (the mutual distance $<<\lambda$ ), they influence each other. In this case, the $Z$ sensor in the $z$ axis creates such electric field component to which the $\mathrm{Y}$ sensor in the $y$ axis is sensitive. This electric field component $\mathrm{E}_{\mathrm{y}}$ corresponds to the relation (6). The $\mathrm{E}_{\mathrm{y}}$ component is tangential to the $\mathrm{Y}$ sensor and creates induced voltage on its terminals. It is assumed that the $\mathrm{Y}$ dipole is loaded by $Z_{s}$. Then the induced voltage on its terminals is calculated due to the relation (4) by using relations (2) and (3). But this way has one non-negligible error - nonhomogeneousness of $\mathrm{E}_{\mathrm{y}}$ electric field component in the place of the Y sensor. By [3] the output voltage of such placed sensor depends on the value $\mathrm{E}_{\mathrm{y}}$ averaged through the $\mathrm{Y}$ dipole length (sensor length)

$$
U_{y}=U_{s L}=h_{e f} \frac{Z_{s}}{Z_{i}+Z_{s}} \frac{1}{2 h} \int_{-h+y}^{h+y} E_{y r}(\xi) d \xi
$$

The meaning of position vectors figuring in (7) is clarified in Fig. 2.

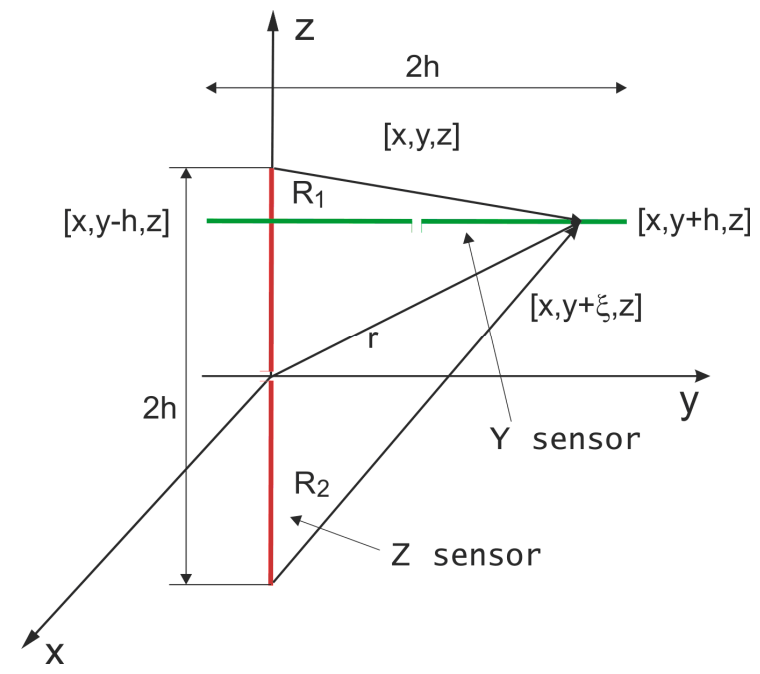

Fig.2. Position vectors of two sensors (dipoles).

The induced voltage on terminals of the loaded $Y$ dipole depends on mutual position of $\mathrm{Z}$ and $\mathrm{Y}$ dipoles and causes the error of electromagnetic field measurement. If assuming two sensors, this error is expressed by

$$
\delta=\frac{\sqrt{U_{z}^{2}+U_{y}^{2}}-U_{z}}{U_{z}} \cdot 100 \%
$$

The relation (8) expresses relative error [7] of electric field measurement. As final consequence, it can help finding such a mutual position of $\mathrm{Z}$ and $\mathrm{Y}$ dipoles, in which their mutual influence can be minimal (zero in ideal case).

\section{RESUltS OF ANALYTICAL SOLUTION}

The existence of homogeneous electromagnetic field is assumed. Its electric vector is oriented in the $z$ axis direction and its intensity is $E_{\mathrm{z}}=1 \mathrm{~V} / \mathrm{m}$ and frequency is $f=1 \mathrm{GHz}$. The electromagnetic field sensor (electric dipole) is oriented into the $z$ axis direction, too. The dipole length $2 \mathrm{~h}$ has such value that relation $\mathrm{k} \cdot \mathrm{h}=\pi / 2$ is valid. The dipole of the arm length $\mathrm{h}=\lambda / 4$ has inner impedance $\mathrm{Z}_{\mathrm{i}}=73+\mathrm{j} 42 \Omega$ [3]. To simplify it, the dipole is loaded with the impedance $Z_{s}$. In case of a complex inner impedance $Z_{i}$ and load impedance $Z_{s}$ is $Z_{i}$ $=\mathrm{Z}_{\mathrm{s}}{ }^{*}$, where $*$ indicates the complex conjugate. The substitution diagram of such a connection is in Fig.3.

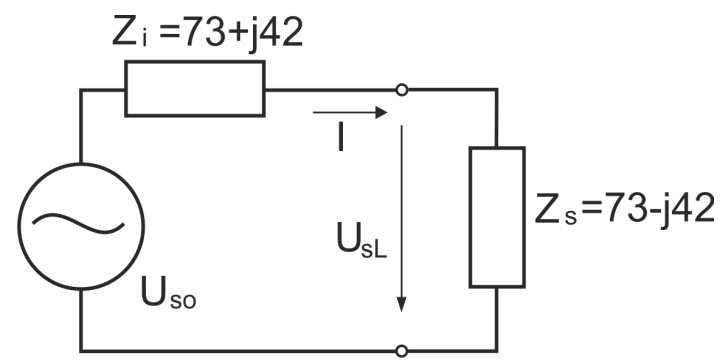

Fig.3. Substitution diagram of the dipole placed in electromagnetic field. 
Based on Fig.3., (5), (3) and (2) the current on terminals of the $\mathrm{Z}$ dipole is calculated

$$
I_{z}=h_{e f} \cdot E_{z} \frac{1}{Z_{i}+Z_{s}}=0.654 \mathrm{~mA}
$$

In the vicinity of the $\mathrm{Z}$ dipole, the current $\mathrm{I}_{\mathrm{z}}$ induces scattered electric field. The scattered electric field vector has components oriented into all directions of the coordinate system. The $Y$ direction is very interesting because the sensor of the $\mathrm{Y}$ dipole is placed there. Through (6), the dependence of the magnitude of the $y$ component of the electric field on distance is demonstrated in Fig.4.

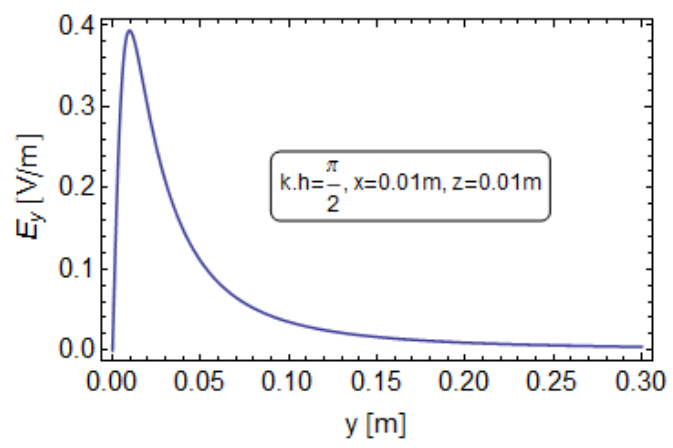

Fig.4. Dependence of the $\mathrm{Z}$ sensor intensity of radiated electric field $\mathrm{E}_{\mathrm{y}}$ on the distance.

As can be seen in Fig.4., the Y sensor placed in the $y$ axis direction in the vicinity of the $\mathrm{Z}$ sensor is affected by the electric field intensity of $0.01-0.4 \mathrm{~V} / \mathrm{m}$. In the $\mathrm{Y}$ sensor, the electric field affects terminal voltage. Its amplitude is calculated through (7). Behaviour of the terminal voltage in dependence on the distance from the centre of the dipole is shown in Fig. 5 .

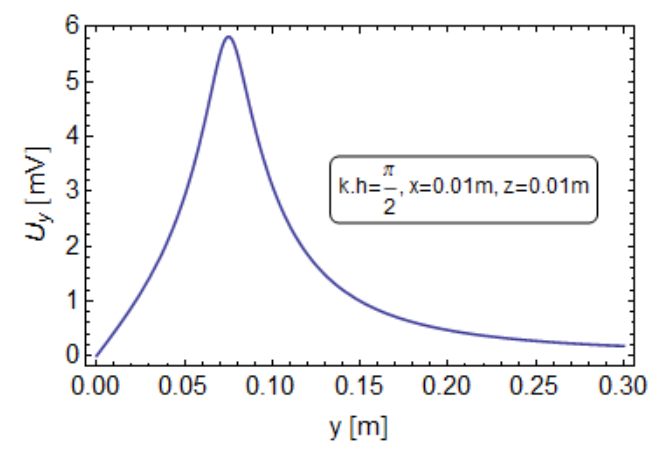

Fig.5. Dependence of the terminal voltage of the Y sensor on the distance from the $\mathrm{Z}$ sensor in the $y$ axis direction.

According to (1), the magnitude of measured electric component is proportional to the total voltage of the probe (of three sensors). Individual voltages of sensors are mathematically processed and thus they form the output voltage on the probe.

In this analyzed case, the voltage $U_{z}$ (it represents magnitude of the incidence field) adds to the voltage $U_{y}$ (it does not represent properties of incidence field) whereby the systematic error in measuring the incidence field intensity arises. To explain this error numerically, the terminal voltage of the loaded $\mathrm{Z}$ dipole from (2) has to be expressed. For the values of the field intensity, the frequency and the $Z$ dipole dimensions, the value of terminal voltage $U_{z}$ of the loaded dipole is:

$$
U_{z}=\frac{2(1-\cos (k h))}{k} E_{i t} \frac{Z_{i}}{Z_{i}+Z_{s}}=47.7 \mathrm{mV}
$$

After inserting the results from Fig.5. and (10) into (8) the dependence of the relative systematic error of the electric field measurement on the mutual position of $\mathrm{Z}$ and $\mathrm{Y}$ sensors is gained.

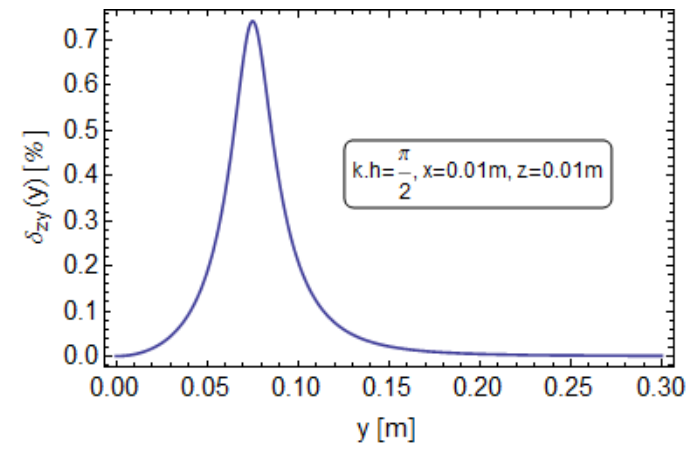

Fig.6. Dependence of the relative systematic error of the electric field measurement on the mutual position of $Z$ and $Y$ sensors.

The mutual vicinity of two sensors introduces the relative error $0-0.8 \%$ into the measurement (Fig.6.). The greatest effect of sensors occurs at the distance of about $0.75 \mathrm{~m}$ which represents the shift of $\lambda / 4$ in the $y$ axis direction.

After the mutual position of transducers is changed in the way that the $Y$ sensor is shifted to the $\mathrm{z}=60 \mathrm{~mm}$ (in the neighborhood of this distance the greatest effect of transducers is assumed), the terminal voltage of loaded sensor is calculated using the equation (7). The result of the analytic calculation is in Fig.7. Comparing Fig.5. to Fig.7., it can be seen that the shape of both curves is the same, only the size of the peak has changed. In this case (Fig.7.), the change is $20 \mathrm{mV}$ at the wavelength frequency of approximately $\lambda / 4$.

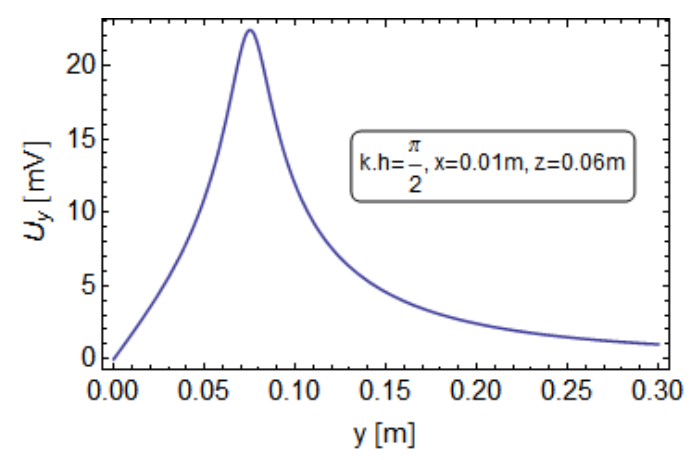

Fig.7. Dependence of the terminal voltage of the Y sensor on the distance from the $\mathrm{Z}$ sensor in the $y$ axis direction. 
As in the previous case in the calculation of the systematic error of measurement, equations (10), (8), and Fig.7. are used.

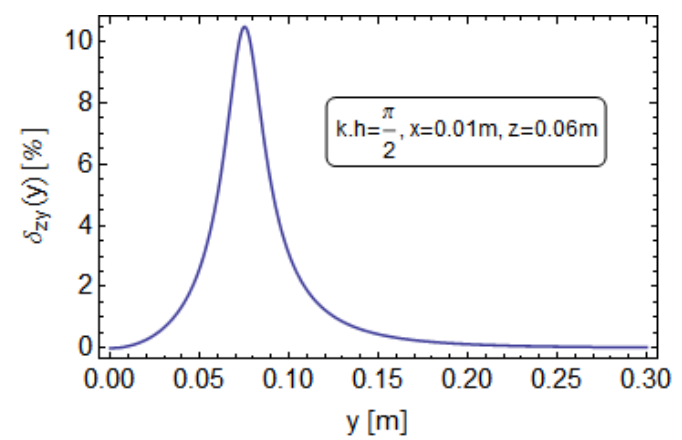

Fig.8. Dependence of the systematic relative error of the measurement of the electric field on mutual position of $\mathrm{Y}$ and $\mathrm{Z}$ sensors.

In this case, the result of the measurement of electromagnetic field is strongly influenced - up to approximately $11 \%$. To make a complex image about the mutual position of sensors and about the relative error, which results from their mutual influence, Table 1. was made.

Table 1. Relative error caused by the mutual influence of sensors. $\mathrm{Z}$ sensor placed on the $z$ axis. Y sensor is parallel to the $y$ axis and moved in $z$ and $y$ axis.

\begin{tabular}{|l|c|c|c|c|c|c|c|c|}
\hline $\mathbf{z}[\mathbf{m m}]$ & 50 & 60 & 70 & 80 & 50 & 60 & 70 & 80 \\
\hline $\mathbf{y}[\mathbf{m m}]$ & 75 & 75 & 75 & 75 & 60 & 60 & 60 & 60 \\
\hline $\boldsymbol{\delta}[\%]$ & 10.2 & 10.4 & 8.3 & 4.6 & 4.9 & 5.0 & 4.2 & 2.9 \\
\hline
\end{tabular}

According to the presented analysis results, the individual sensor transducers must have such mutual distance that they avoid $\lambda / 4$ position of its resonant frequency - in all directions of the coordinate system (this assumes that one sensor is placed on the $\mathrm{z}$ axis). During the electromagnetic field measurements using several probes, engineers in laboratories and test chambers have to follow a similar rule.

\section{NUMERICAL SOLUTION}

To verify the correctness of the analytic method of the calculation either the measurement or the numeric simulation of the characteristics of electromagnetic field probes is necessary. In this case, it is difficult to perform the real experiment because the result of the measurement is negatively influenced by the measuring method itself. Consequently, to verify the analytic method, the numeric one is used. Method of Moment (MoM), represented by the software system FEKO, appears to be the most convenient type of the method [6]. The first task of the numeric simulation is to calculate the induced voltage on the terminals of loaded dipole for the same parameters as defined in the beginning of Chapter 4 (length of dipole $2 \mathrm{~h}=150 \mathrm{~mm}$, diameter of dipole $\mathrm{d}=0.001 \mathrm{~mm}$ and the incident frequency $\mathrm{f}=1 \mathrm{GHz}$ ). Dipole is placed in infinitely spacious medium in the $z$ axis direction. Homogeneous electromagnetic field with the intensity of $E_{z}=1 \mathrm{~V} / \mathrm{m}$ influences the dipole as it is indicated in Fig.8. In the simulation process the dipole was divided into 31 segments with perfect conducting material, where segment number 11 was loaded by impedance $Z_{s}=73-\mathrm{j} 42 \Omega$. The segment length must satisfy condition: $1<\lambda / 10$. The boundary conditions of the numerical model are included in the method (MoM) of solution (MoM solve the Pocklington equation).

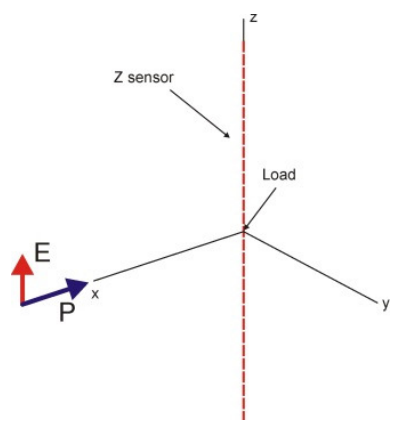

Fig.9. FEKO one dipole model.

Using this configuration of the dipole and electromagnetic field, direction is calculated by the current that flows through the load

$$
I_{L}=0.6538 m A
$$

This current value is nearly identical to the analytically calculated current. It means that the relations (2), (3), (4), and (5) are derived correctly.

In the next step, another sensor - the dipole Y, parallel to the $y$ axis (Fig.9.) - was added to the model in Fig.8.

The induced voltage on the load of the second dipole (parallel to the $y$ axis) was calculated for the shift of the dipole in its y axis direction. See Fig.11. for the results.

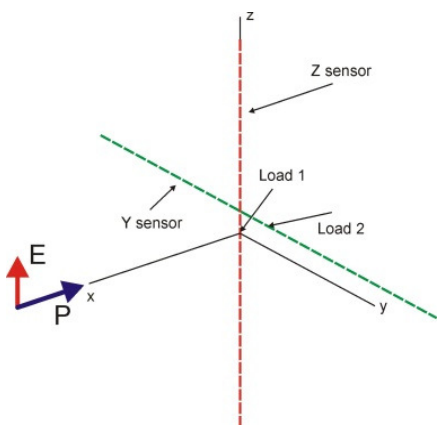

Fig.10. FEKO model of the perpendicular dipoles.

Comparing Fig.11. to Fig.5., we can point out that both courses are practically identical, with the peak value of nearly $6 \mathrm{mV}$. The difference between these two figures is in the width of the curves and the displacement of the peaks of the curves. 


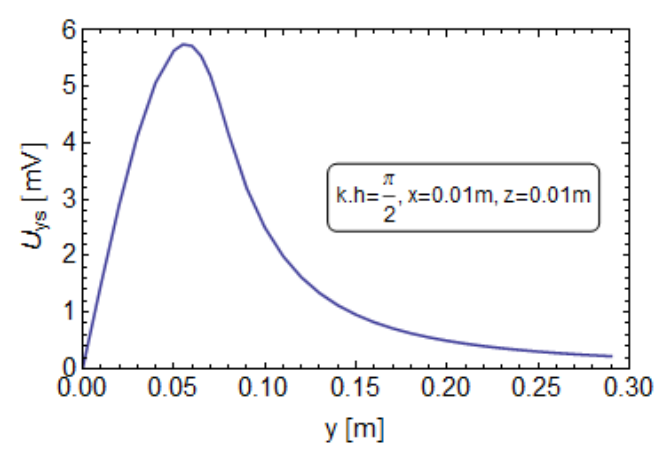

Fig.11. Dependence of the terminals voltage of the $\mathrm{Y}$ dipole (sensor) on the distance from the $\mathrm{Z}$ dipole (sensor) in the $y$ axis direction - numerical calculation.

When the analytical calculation was used, the diameter of the dipole was considered to be zero. In the numerical calculation the diameter of the dipole was $d=0.001 \mathrm{~mm}$. At the first sight, this negligible diameter of dipole causes the reduction of its quality and the enlargement of its wavelength. This is what explains the differences between Fig.5. and Fig. 11.

The Y dipole was moved to the distance $\mathrm{z}=0.06 \mathrm{~m}$ and $\mathrm{a}$ series of simulations of induced terminal voltage was conducted. The dipole was loaded by the impedance $Z=73$ $-\mathrm{j} 42 \Omega$ and shifted to the $y$ axis direction. The result of the simulation is in Fig. 12.

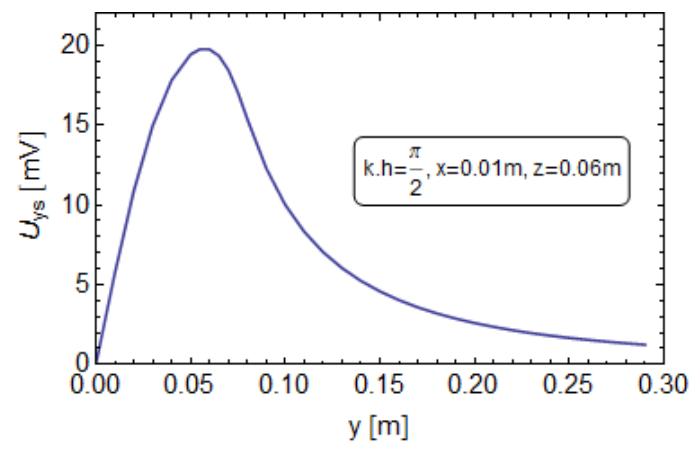

Fig.12. Dependence of the terminal voltage of the $\mathrm{Y}$ dipole (sensor) on the distance from the $\mathrm{Z}$ dipole (sensor) in the $y$ axis direction - numerical calculation.

In this case, the peak value of the curve was also almost the same as it was obtained by the analytical calculation about $20 \mathrm{mV}$. The conclusions here and in the numerical calculation are the same (Fig.12.).

In spite of the mentioned facts, results of the numerical simulations show excellent agreement of both, analytical and numerical calculations. The assumptions presented in [3] and the validity of the relation (7) was verified.

\section{CONCLUSION}

The aim of this article was to show how to calculate the interactive influence of the electric field sensors that are probed together. Half-wave dipoles were used as sensors. Only two electric field sensors were used to simplify the situation and ease the verification of analytical calculations.
The field sensors were perpendicular to each another. In spite of the intuitive assumption that the sensors would not influence themselves interactively, both results of analytical calculation and numeric simulation confirmed the opposite. The mutual affection of sensors depends on their mutual position, and in the worst case causes the measurement error of the field up to $11 \%$.

The use of the half-wave dipoles and their location in the area is a rare practice but it gives the idea about the maximum errors of the dipole. By using the probes based on the short electric or resistive dipoles (mutually perpendicular placed in space), the error of measuring will approach up to $11 \%$ only as a limit.

In the case of the layout of individual sensors of the probe, the results of this article are universal. For all types of used sensors, the mutual affection is guided by Table 1 . Therefore, for the construction of electromagnetic probes the following recommendations are necessary to be respected:

- The sensors have to be placed in such a way that the shift of their centers avoids $\lambda / 4$ of the wavelength of their maximum frequency;

- It is convenient to place sensors so that their centers create the origin of the coordinate system;

- If it is not possible to perform the previous recommendation then it is convenient to place the transducers so that any mutual projection can exist.

The results presented in this article could aid in the construction of the electromagnetic field probes made on the basis of the resonant, short electric and resistive dipoles.

There exist also the other space arrangements of dipoles in probe of the electromagnetic field. There exist also the other frequencies of incident field which influence their mutual impedance. The complexity of this problem is outside the scope of this paper. From the obtained results we can assume that the gained results represent the worst case scenario out of all combinations of mutually perpendicular dipoles (sensors).

\section{ACKNOWLEDGMENT}

This work was supported by the Slovak Research and Development Agency under the contract No. APVV-033311 and by the project VEGA 1/0963/12.

\section{REFERENCES}

[1] Vavra, Š., Turán, J. (1989). Antény a šírenie elektromagnetických vín. Bratislava: Alfa.

[2] Balanis, A.C. (1997) Antenna Theory Analysis and Design, 3th edition. John Wiley \& Sons.

[3] Slížik, J., Hart'anský, R. (2013). Metrology of electromagnetic intensity measurement in near field metrology of electromagnetic intensity measurement in near field. Quality Innovation Prosperity, 17 (1), 57-66.

[4] Hart’anský, R., Slížik, J., Maršálka, L. (2013). Dipole near field analysis - a closed form calculation in cartesian coordinates. Journal of Electrical Engineering, 64 (5), 1-4. 
[5] Bittera, M., Smieško, V., Kováč, K. (2012). Modified uncertainty estimation of antenna factor measurement by standard site method. Measurement, 45 (2), 190198.

[6] Kamenský, M., Kováč, K., Války, G. (2012). Improvement in spectral properties of quantization noise of harmonic signal using multiresolution quantization. IEEE Transactions on Instrumentation and Measurement, 61 (11), 2888-2895.
[7] Roller, C., Wasylkiwskyj, W. (1992). Effects of mutual coupling on super-resolution DF in linear arrays. In IEEE International Conference on Acoustics, Speech, and Signal Processing, ICASSP-92. IEEE, Vol. 5, 257-260.

[8] Božek, P., Pokorný, P. (2014). Analysis and evaluation of differences dimensional products of production system. Applied Mechanics and Materials, 611, 339345.

Received March 10, 2014 Accepted October 31, 2014. 